\title{
HARMONIC ANALYSIS ON THE QUANTIZED RIEMANN SPHERE
}

\section{JAAK PEETRE and GENKAI ZHANG}

\author{
Matematiska institutionen \\ Stockholms universitet \\ Box 6701 \\ S-113 85 STOCKHOLM \\ Sweden
}

(Received Febr. 12, 1992)

\begin{abstract}
We extend the spectral analysis of differential forms on the disk (viewed as the non-Euclidean plane) in recent work by J. Peetre - L. Peng - G. Zhang to the dual situation of the Riemann sphere $S^{2}$. In particular, we determine a concrete orthogonal base in the relevant Hilbert space $L^{\nu, 2}\left(S^{2}\right)$, where $-\frac{\nu}{2}$ is the degree of the form, a section of a certain holomorphic line bundle over the sphere $S^{2}$. It turns out that the eigenvalue problem of the corresponding invariant Laplacean is equivalent to an infinite system of one dimensional Schrödinger operators. They correspond to the Morse potential in the case of the disk. In the course of the discussion many special functions (hypergeometric functions, orthogonal polynomials etc.) come up. We give also an application to "Ha-plitz" theory.
\end{abstract}

KEYWORDS AND PHRASES: Riemann sphere, quantization, reproducing kernel, invariant Cauchy-Riemann operator, invariant Laplacean, Morse operator, Hankel operator, hypergeometric function, orthogonal polynomial 1980 AMS SUBJECT CLASSIFICATION: 30C40, 47B35

\section{INTRODUCTION.}

We wish to extend the considerations in a recent paper [10] valid for the unit disk $D$ or, equivalently, the upper halfplane $U$ (regarded as a model of the non-Euclidean (or Lobachevskiu) plane) to the case of the Riemann sphere $S^{2}$. That is, we shall pass to the dual symmetric space.

Let us briefly recapitulate the main contents of [10]. There we studied certain weighted $L^{2}$ spaces over $D$ depending on a parameter $\alpha>-1$ (in the physical interpretation $\frac{1}{\alpha+2}$ is Planck's constant) and described their orthogonal decomposition under the action of the Moebius group $\mathrm{SU}(1,1)$. This decomposition was found using a certain invariant Laplacean. Special attention was payed to the discrete spectrum. In particular, explicit orthogonal bases in terms of hypergeometric functions were found in each of the irreducible discrete parts in the decomposition (each isomorphic to a space in the holomorphic discrete series). The orthogonal bases were then used to study "Ha-plitz" type linear operators acting between these spaces (boundedness, compactness, Schatten-von Neumann properties).

In this paper we, thus, carry over the same program to the case of the sphere. A major difference is now that the corresponding invariant Laplacean has only discrete spectrum. Otherwise the presentation is remarkably parallel. Again lots of interesting special functions (hypergeometric and other) arise throughout the discussion, which in fact has been part of the motivation for undertaking this study. We further elucidate several points in the hyperbolic case left over in [10], for instance the rather mysterious appearance of the Morse potential in the parallel treatment in [3].

We should also say what we intend by the word "quantization" (occurring in the title). We use it mainly in the sense of Berezin [1] (cf. [8]). Thus we view quantization as a theory of deformation of line bundles, and the relevant Hilbert spaces, arena for the operator theory involved, arise really as spaces of sections of these line bundles.

Most of the results of this paper should extend rather easily to the case of $\mathbf{P}^{n}$ (complex projective space, which is the compact counterpart of the complex unit ball ${ }^{1}$ ). Of course, all this is just a tiny portion of a vast program and we expect similar results to be true for arbitrary Hermitean symmetric spaces, both compact and non-compact ones. 
The plan of the paper is as follows. In Section 1 we uncover the structure of the eigenspaces of the invariant Laplacean, in particular we find an explicit orthogonal basis in them. A novelty is perhaps that we determine the spectrum factoring suitable functions of the Laplacean. In fact, the same thing can be done on any Riemann surface so we have deferred the details of the proof to an appendix (as a Section 6). In Section 2 we use the orthogonal basis from Section 1 to write down the reproducing kernels involved. Some applications in the spirit of Vilenkin's great book [12] are given in Section 3. Many more such applications are possible! In Section 4 some operator theory consequences of the previous discussion are briefly indicated. Lastly, in Section 5 we return to the basis vectors and consider the differential equations satisfied by them. In particular, we write down the "periodic" analogue of the Morse operator.

\section{THE ORTHOGONAL BASIS.}

We shall work within a fixed coordinate neighborhood with coordinate $z$ obtained by deleting one point $\infty$ ("the point at infinity"). In other words, $S^{2}$ will be identified with the extended complex plane $\hat{\mathbf{C}}=\mathbf{C} \cup\{\infty\}$ (or the complex projective line $\mathbf{P}^{1}$ ). Near $\infty$ we use instead of $z$ the coordinate $\frac{1}{z}$.

The essential change compared to $[10]$ is that the factor $1-|z|^{2}$, everywhere, has to be replaced by $1+|z|^{2}$ (and $1-z \bar{w}$ by $\left.1+z \bar{w}\right){ }^{2}$ Thus, the invariant Cauchy-Riemann operator is given by

$$
\bar{D}=\left(1+|z|^{2}\right)^{2} \frac{\partial}{\partial \bar{z}}
$$

Similarly, the invariant Laplacean is

$$
\Delta_{\nu}=-\left(1+|z|^{2}\right)^{2} \frac{\partial^{2}}{\partial z \partial \bar{z}}+\nu\left(1+|z|^{2}\right) \bar{z} \frac{\partial}{\partial \bar{z}}
$$

The relevant group is now the compact group SU(2), consisting of all $2 \times 2$ complex matrices $\xi=\left(\begin{array}{ll}a & b \\ c & d\end{array}\right)$ such that $a d-b c=1, c=-\bar{b}, d=\bar{a}$, and the action we have in mind is

$$
U_{\xi}^{(\nu)}: f(z) \mapsto f(\xi(z))\left(\xi^{\prime}(z)\right)^{-\frac{\nu}{2}}=f\left(\frac{a z+b}{c z+d}\right)(c z+d)^{\nu} .
$$

In this notation we have

$$
\bar{D} U^{(\nu)}=U^{(\nu+2)} \bar{D}
$$

Notice that, compared to [10], we have changed $\nu$ to $-\nu$ in the last formula. Also we are going to assume that $\nu$ is an integer $\geq 0$. This is very convenient, as we are going to work with the Hilbert space $L^{2, \nu}\left(S^{2}\right)$ of functions with the metric

$$
\|f\|^{2}=\int_{\mathbf{C}}|f(z)|^{2} \frac{d \mathbf{a}(z)}{\left(1+|z|^{2}\right)^{\nu+2}}
$$

where $d \mathbf{a}$ is the normalized area measure, $d \mathbf{a}(z)=\frac{d x d y}{\pi}$.

REMARK. The measure $\frac{d \mathbf{a}(z)}{\left(1+|z|^{2}\right)^{\nu+2}}$ is sometimes named after various authors: Berezin, Bergman, Dzhrabshyan, Harish-Chandra, Kostant etc. From a highbrow point of view we should, mitean holomorphic line bundle over $S^{2}$ (differential not as functions but as sections of an HerPicard group of $\mathbf{P}^{1}$ is isomorphic to $\mathbf{Z}$, so the holom forms of degree $-\frac{\nu}{2}$ ). In fancy language, the discrete parameter $\nu$. If we interpret the for holomorphic line bundles over $\mathbf{P}^{1}$ are labeled by a the family $\left\{(c z+d)^{\nu}\right\}$ gives the transition formula $w=\frac{a z+b}{c z+d}$ as a change of coordinates on $\mathbf{P}^{1}$,

The subspace of analytic function in $L^{2, \nu}\left(S^{2}\right)$ will be denoted by $A^{2, \nu}$. It is finite dimensional and consists precisely of all polynomials of degree $\leq \nu$, so that $\operatorname{dim} A^{2, \nu}=\nu+1$. This gives the eigenspace of $\Delta_{\nu}$ corresponding to the lowest ("zeroth") eigenvalue 0 . To describe the other $(l+1)$-st power $\bar{D}^{l+1}$ following the same pattern as in [10], first analyze the nullspace of the $(l+1)$-st power $\bar{D}^{l+1}$ of $\bar{D}$. We denote this space by $B_{l}, B_{l}=\operatorname{ker} \bar{D}^{l+1} \cap L^{2, \nu}\left(S^{2}\right)$. The $l$-th 
eigenspace $A_{l}^{2, \nu}(l=0,1,2, \ldots)$, with the eigenvalue $l(\nu+1+l)$, is obtained as the orthogonal complement of $B_{l-1}$ in $B_{l}$,

$$
A_{l}^{2, \nu}=B_{l} \ominus B_{l-1} \quad\left(B_{-1}=0\right) .
$$

This follows from the following lemma.

Lemma 1. Let $D_{\nu}: L^{2, \nu+2} \rightarrow L^{2, \nu}$ be the adjoint of $\bar{D}=\bar{D}_{\nu}$ considered as an operator from $L^{2, \nu}$ into $L^{2, \nu+2}$, that is,

$$
D_{\nu}=-\frac{\partial}{\partial z}+(\nu+2) \frac{\bar{z}}{1+|z|^{2}}
$$

Then we have the factorization

$$
D_{\nu} D_{\nu+2} \ldots D_{\nu+2 l} \bar{D}^{l+1}=\Delta_{\nu}\left(\Delta_{\nu}-(\nu+2)\right)\left(\Delta_{\nu}-2(\nu+3)\right) \ldots\left(\Delta_{\nu}-l(\nu+1+l)\right) .
$$

Its proof relies on the following identity:

$$
\bar{D} D_{\nu}=D_{\nu+2} \bar{D}-(\nu+2) \text {. }
$$

For details see the Appendix, where the proof is given in the context of an arbitrary Riemann surface equipped with a metric with positive Gaussian curvature.

Now we begin to uncover the structure of the space $B_{l}$.

LEMMA 2. Every global solution of the differential equation $\bar{D}^{l+1} f=0$ has the unique representation

$$
f=\sum_{j=0}^{l} g_{j} \frac{\bar{z}^{j}}{\left(1+|z|^{2}\right)^{\jmath}}
$$

where each $g,(j=0,1, \ldots, l)$ is a polynomial of degree $\leq \nu+2 l$. However, the $l-j$ highest coefficients of $g_{j}$ are determined by $g_{l}, \ldots, g_{j+1}$. In particular, the dimension of $B_{l}$ is $(l+1)(\nu+$ $l+1)$.

PROOF: That every solution of $\bar{D}^{l+1}$ has the representation (1) with $g$, entire in $z$ follows as in [10] solving the differential equation. In order to see which are the restrictions on $g$, at $\infty$ we make the change of variable $z \mapsto \frac{1}{z}$. Then we find

$$
\begin{aligned}
f & =\sum_{j=0}^{l} g_{j}\left(\frac{1}{z}\right) \frac{\left(\frac{1}{\bar{z}}\right)^{j}}{\left(1+\frac{1}{|z|^{2}}\right)^{j}} z^{\nu}= \\
& =\sum_{j=0}^{l} g_{j}\left(\frac{1}{z}\right) z^{\nu+\jmath}\left(1-\frac{z \bar{z}}{1+|z|^{2}}\right)^{j}= \\
& =\sum_{j=0}^{l} g_{\jmath}\left(\frac{1}{z}\right) z^{\nu+\jmath} \sum_{r=0}^{J}(-1)^{r}\left(\begin{array}{l}
r \\
j
\end{array}\right) \frac{z^{r} \bar{z}^{r}}{\left(1+|z|^{2}\right)^{r}}= \\
& =\sum_{r=0}^{l}(-1)^{r}\left(\sum_{j=r}^{l}\left(\begin{array}{l}
r \\
j
\end{array}\right) g_{\jmath}\left(\frac{1}{z}\right) z^{\nu+\jmath+r}\right) \frac{\bar{z}^{r}}{\left(1+|z|^{2}\right)^{r}} .
\end{aligned}
$$

For instance, the $l$-th coefficient in the outer sum is, up to sign, just $g_{l}\left(\frac{1}{z}\right) z^{\nu+2 l}$. It follows that $g_{l}$ must be a polynomial of degree $\leq \nu+2 l$. Similarly, the $(l-1)$-st coefficient is, again up to sign,

$$
\lg _{l}\left(\frac{1}{z}\right) z^{\nu+2 l-1}+g_{l-1}\left(\frac{1}{z}\right) z^{\nu+2 l-2} .
$$


It follows that $g_{l-1}$ must be a polynomial of degree $\leq \nu+2 l-1$ and that $l \hat{g}_{l}(\nu+2 l)+\hat{g}_{l-1}(\nu+2 l-1)=$ 0 . (We use the sign “ to denote Taylor coefficients.) The general statement about the functions $g$, follows now readily by induction. It is now likewise clear that

$$
\begin{aligned}
\operatorname{dim} B_{l} & =\nu+2 l+1+(\nu+2 l-2+1)+\cdots+(\nu+2+1)+(\nu+0+1)= \\
& =(l+1)(\nu+1)+2(l+l-1+\cdots+1+0)= \\
& =(l+1)(\nu+1)+2 \cdot \frac{(l+1) l}{2}=(l+1)(\nu+l+1) .
\end{aligned}
$$

Next, we decompose the space $B_{l}$ under the action of the rotation group $\mathrm{SO}(2)$ (the isotropy group of the origin $z=0$ ). The elements of the $n$th space in this decomposition must be of the form

$$
f=z^{n} q\left(\frac{|z|^{2}}{1+|z|^{2}}\right)
$$

where $q=q(t)$ is a polynomial of degree $\leq l$. If $n<0$ it must vanish to the order $-n$ at the origin $t=0: q(t)=\mathrm{O}\left(t^{-n}\right)$. Similarly, if $n>\nu$ it must vanish to the order $n-\nu$ at the point $t=1: q(t)=\mathrm{O}\left((1-t)^{n-\nu}\right)$. The last statement follows again by making the substitution $z \mapsto \frac{1}{z}$. We then get

$$
f=z^{\nu-n} q\left(1-\frac{z \bar{z}}{1+|z|^{2}}\right) .
$$

It follows that we must have $-l \leq n \leq l+\nu$.

Let us now compute the norm of a function of the form (2):

$$
\begin{aligned}
\|f\|^{2} & =\iint_{\mathbf{C}}|z|^{2 n}\left|q\left(\frac{|z|^{2}}{1+|z|^{2}}\right)\right|^{2} \frac{d x d y}{\pi\left(1+|z|^{2}\right)^{\nu+2}}= \\
& =\int_{0}^{\infty} \int_{0}^{2 \pi} r^{2 n}\left|q\left(\frac{r^{2}}{1+r^{2}}\right)\right|^{2} \frac{r d r d \phi}{\pi\left(1+r^{2}\right)^{\nu+2}}= \\
& =\int_{0}^{\infty} r^{2 n}\left|q\left(\frac{r^{2}}{1+r^{2}}\right)\right|^{2} \frac{r d r}{\pi\left(1+r^{2}\right)^{\nu+2}}= \\
& =\int_{0}^{\infty}|q(t)|^{2} t^{n}(1-t)^{\nu-n} d t=\|q\|^{2} .
\end{aligned}
$$

Here we have introduced polar coordinates, writing $z=r e^{i \phi}$, and put

$$
t=\frac{|z|^{2}}{1+|z|^{2}}=\frac{r^{2}}{1+r^{2}} \quad \text { with } 1-t=\frac{1}{1+|z|^{2}}=\frac{1}{1+r^{2}}, \quad d t=\frac{2 r d r}{\left(1+r^{2}\right)^{2}}
$$

Now we orthogonalize the set of all polynomials in the metric $\|q\|$. We get then for each integer $n$ a set of orthogonal polynomials $\left\{q_{l_{n}}(t)\right\}$. If we keep $n$ fixed and vary instead $l$ we get an orthogonal basis for the $l$-th eigenspace $A_{l}^{2, \nu}$ of the operator $\Delta_{\nu}$ of the form $\left\{e_{n l}(z)\right\}$, where $-l \leq n \leq l+\nu$. It will be convenient to set

$$
e_{n l}(z)=z^{n} q_{l n}\left(\frac{|z|^{2}}{1+|z|^{2}}\right) .
$$

In particular, we see that $\operatorname{dim} A_{l}^{2, \nu}=\nu+2 l+1$ in agreement with Lemma 2. Namely, this lemma shows that

$$
\operatorname{dim} A_{l}^{2, \nu}=\operatorname{dim} B_{l}-\operatorname{dim} B_{l-1}=(l+1)(\nu+l+1)-l(\nu+l)=\nu+(l+1)^{2}-l^{2}=\nu+2 l+1 .
$$

REMARK. It is convenient to use also instead of $t$ the parameter $t_{1}=2 t-1=\frac{|z|^{2}-1}{|z|^{2}+1}$; then $1+t_{1}=2 t, 1-t_{1}=2(1-t), d t_{1}=d t$. If $t$ ranges from 0 to 1 , then $t_{1}$ ranges from -1 to 1 . So if we write $Q\left(t_{1}\right)=q(t)$, we get the metric (apart from a constant factor $2^{\nu+1}$ )

$$
\|Q\|_{1}^{2}=\int_{-1}^{1}\left|Q\left(t_{1}\right)\right|^{2}\left(1+t_{1}\right)^{n}\left(1-t_{1}\right)^{\nu-n} d t_{1}
$$

Thus if $0 \leq n \leq \nu$ we have the conventional Jacobi polynomials $P_{l}^{(n, \nu-n)}$ (up to normalizing factors). If we agree to use the same notation also in the general case, we can write our basis as 


$$
\left\{z^{n} P_{l}^{(n, \nu-n)}\left(\frac{|z|^{2}-1}{|z|^{2}+1}\right)\right\}
$$

where as before $-l \leq n \leq l+\nu$. A possibly even better parameter than $t_{1}$ is $t_{2}=1-t_{1}=1-2 t=$ $\frac{1-|z|^{2}}{1+|z|^{2}}$. If we realize $S^{2}$ as the standard sphere in $\mathbf{R}^{3}$ (using stereographic projection onto $\hat{\mathbf{C}}$ ), it gets an immediate geometric meaning. Namely, if we let $\theta, \phi$ be polar coordinates about the north pole, we can write $z=\tan \frac{\theta}{2} e^{\imath \phi}$. It follows then that, indeed, $t_{2}=\cos \theta$.

It should be clear that our result generalizes the classical Laplace series (expansion in spherical harmonics). This is the case $\nu=0$, when the elements are genuine functions, not sections of a line bundle. For instance, it is well-known that the $l$ th eigenvalue of the Laplace-Beltrami operator equals $l(l+1)$, which agrees with our formula.

Let us study the polynomials $q_{l n}$.

Lemma 3. If properly normalized the polynomials $q_{l n}, l \geq \max (-n, n-\nu)$, are given by a Rodrigues's formula:

$$
q_{\ln }(t)=t^{-n}(1-t)^{n-\nu}\left(\frac{d}{d t}\right)^{l}\left[t^{n+l}(1-t)^{\nu-n+l}\right]
$$

and

$$
\left\|q_{l n}\right\|^{2}=\int_{0}^{1}\left|q_{l n}(t)\right|^{2} t^{n}(1-t)^{\nu-n} d t=\frac{l !(n+l) !(\nu-n+l) !}{(2 l+\nu+1)(\nu+l) !} .
$$

Before passing to its proof, let us introduce some notation. We define the "ascending" factorial (or Pochhammer symbol)

$$
(a)_{n}=a(a+1) \ldots(a+n-1)
$$

and also the "descending" factorial

$$
(a)_{n}^{-}=a(a-1) \ldots(a-n+1)=(-1)^{n}(-a)_{n} .
$$

Clearly $\left(\frac{d}{d t}\right)^{n} t^{a}=(a)_{n}^{-} t^{a-n}$. Recall further the useful formula

$$
(a)_{n}=(a)_{l}(-1)^{n-l}(n-1-a)_{n-l},
$$

which will repeatedly be used in what follows.

Now we proceed with the proof of Lemma 3.

Proof: By Leibnitz's formula

$$
\begin{aligned}
q_{l n}(t) & =t^{-n}(1-t)^{n-\nu} \sum_{h=0}^{l}\left(\begin{array}{l}
l \\
h
\end{array}\right)(n+l)_{l-h}^{-}(\nu+l-n)_{h}^{-} \times \\
& \times t^{n+l-l+h}(-1)^{n}(1-t)^{\nu-n+l-h}= \\
& =\sum_{h=0}^{l}(-1)^{h}\left(\begin{array}{l}
l \\
h
\end{array}\right)(n+l)_{l-h}^{-}(\nu+l-n)_{h}^{-} t^{h}(1-t)^{l-h},
\end{aligned}
$$

so it is clear that $q_{l n}$ is a polynomial of degree $\leq l$.

To see that it is exactly of degree $l$ we look at the top coefficient. Expand the expression in (3) in a power series in $t$. Then we see that for large $t$ the main contribution to the derivation comes from the term

$$
\begin{aligned}
& t^{-n}(-1)^{n-\nu} t^{n-\nu}\left(\frac{d}{d t}\right)^{l}\left[t^{n+l}(-1)^{\nu-n+l} t^{\nu-n}\right]= \\
= & (-1)^{l} t^{-\nu}\left(\frac{d}{d t}\right)^{l} t^{2 l+\nu}=(-1)^{l} t^{-\nu}(2 l+\nu)_{l}^{-} t^{2 l+\nu-l}=(-1)^{l}(l+\nu+1)_{l} t^{l} .
\end{aligned}
$$

Thus the top coefficient is $\neq 0$ and equals $(-1)^{l}(l+\nu+1)$. If $n<0$, we have

$$
(n+l)_{l-h}^{-}=(n+l)(n+l-1) \ldots(\overbrace{n+l-(l+n)}^{0}) \ldots(n+l-(l-h)-1)=0,
$$
provided $l-h-1 \geq l+n$ or $h \leq-n-1$. That is $q_{\ln }(t)=\mathrm{O}\left(t^{-n}\right)$. If $n>\nu$, we find in the same
way $q_{\ln }(t)=\mathrm{O}\left((1-t)^{n-\nu}\right)$.

Let us consider the integral 


$$
\left(q_{l n}, t^{3}\right)=\int_{0}^{1} t^{-n}(1-t)^{n-\nu}\left(\frac{d}{d t}\right)^{l}\left[t^{n+l}(1-t)^{\nu-n}\right] \cdot t^{3} d t .
$$

If $j<l$ we can integrate by parts $j+1$ times and each time the term integrated out clearly vanishes. So the integral is 0 . We conclude that

$$
\left(q_{l n}, q_{n \jmath}\right)=0 \quad \text { for } l \neq j
$$

To find the norm, we consider the integral

$$
\left(q_{\ln }, t^{l}\right)=\int_{0}^{1}\left(\frac{d}{d t}\right)^{l}\left[t^{n+l}(1-t)^{\nu-n+l}\right] t^{l} d t
$$

This time we can integrate by parts $l$ times only to produce the integral

$$
\begin{aligned}
& (-1)^{l} l ! \int_{0}^{1} t^{n+l}(1-t)^{\nu-n+l} d t= \\
= & (-1)^{l} l ! \frac{\Gamma(n+l+1) \Gamma(\nu-n+l+1)}{\Gamma(n+l+1+\nu-n+l+1)}=(-1)^{l} \frac{l !(n+l) !(\nu-n+l) !}{(\nu+2 l+1) !} .
\end{aligned}
$$

It follows that

$$
\begin{aligned}
& \left\|q_{l n}\right\|^{2}=(-1)^{l}(l+\nu+1)_{l}\left(q_{l n}, t^{l}\right)= \\
= & (l+\nu+1)_{l} \frac{l !(n+l) !(\nu-n+l) !}{(\nu+2 l+1) !}=\frac{l !(n+l) !(\nu-n+l) !}{(\nu+2 l+1)(\nu+l) !} .
\end{aligned}
$$

Lемма 4. If $n \geq 0$ it is possible to express $q_{l n}$ in terms of the hypergeometric function:

$$
q_{\ln }(t)=(n+1)_{l} F(l+\nu+1,-l ; n+1 ; t) .
$$

We recall that

$$
F(a, b ; c ; z)={ }_{2} F_{1}(a, b ; c ; z)=\sum_{n=0}^{\infty} \frac{(a)_{n}(b)_{n}}{n !(c)_{n}} z^{n} .
$$

Proof: We rewrite the coefficient in (4) as

$$
\begin{aligned}
& \left(\begin{array}{l}
l \\
n
\end{array}\right)(n+l)_{l-h}^{-}(\nu+l-n)_{h}^{-}= \\
= & (-1)^{h} \frac{(-l)_{h}}{h !} \cdot \frac{(n+1)_{l}}{(n+1)_{h}} \cdot(-1)^{h}(n-\nu-l+1)_{h}= \\
= & (n+1)_{l} \frac{(-l)_{h}(n+1)_{l}}{h !(n+1)_{h}} .
\end{aligned}
$$

It follows that

$$
q_{\ln }(t)=(n+1)_{l}(1-t)^{l} F\left(n-\nu-l,-l ; n+1 ; \frac{t}{t-1}\right) .
$$

On the other hand, we know, quite generally, that ([6], bottom of page 8)

$$
F(a, b ; c ; z)=(1-z)^{-b} F\left(b, c-a ; c ; \frac{z}{z-1}\right) .
$$

So that taking $a=n-\nu-l, b=-l, c=n+1, z=t$ we find

$$
q_{l n}(t)=(n+1)_{l} F(l+\nu+1,-l ; n+1 ; t) \text {. }
$$

REMARK. If $\nu \geq n$ we get instead

$$
q_{\ln }(t)=(-1)^{l}(\nu-n+1)_{l} F(l+\nu+1,-l ; \nu-n+1 ; 1-t) .
$$

This is in full agreement with [6], top line of page $9 .^{3}$

\section{THE REPRODUCING KERNEL.}

We can now determine the reproducing kernel $K_{l}(z, w)$ of the space $A_{l}^{2, \nu}$. As $\left\{e_{n l}(z)\right\},-l \leq$ $n \leq \nu+l$, is an orthogonal basis, we have by general facts 


$$
K_{l}(z, w)=\sum_{n=-l}^{\nu+l} e_{n l}(z) e_{n l}(w)\left\|e_{n l}\right\|^{-2}
$$

On the other hand, from the SU(2)-invariance it follows that we must have

$$
K_{l}(z, w)=(1+z \bar{w})^{\nu} \chi\left(\frac{|z-w|^{2}}{\left(1+|z|^{2}\right)\left(1+|w|^{2}\right)}\right),
$$

where $\chi$ is a function of one variable.

To determine $\chi$ it suffices to take $w=0$. Then (5) gives

$$
K_{l}(z, 0)=e_{0 l}(z)\left\|e_{0 l}\right\|^{-2} l !,
$$

as

$$
e_{n l}(0)= \begin{cases}l !, & n=0 \\ 0, & n \neq 0\end{cases}
$$

(If $n>0(6)$ is obvious; if $n<0$ it follows from the fact that $e_{n l}(z)=z^{n} q_{l n}\left(\frac{|z|^{2}}{1+|z|^{2}}\right)$ and $q_{l n}(t)=\mathrm{O}\left(t^{-n}\right)$, so that $e_{n l}(z)=\mathrm{O}\left(|z|^{-n}\right)$.) From Lemma 2 and Lemma 3 (with $n=0$ ) we get

$$
\begin{aligned}
K_{l}(z, 0) & =l ! F\left(l+\nu+1,-l ; 1 ; \frac{|z|^{2}}{1+|z|^{2}}\right)\left(\frac{l !(\nu+l) !}{(2 l+\nu+1)(l+\nu) !}\right)^{-1} \\
& =(2 l+\nu+1) \cdot F\left(l+\nu+1,-l ; 1 ; \frac{|z|^{2}}{1+|z|^{2}}\right) .
\end{aligned}
$$

Thus we draw the following conclusion.

THEOREM 1. The reproducing kernel in $A_{l}^{2, \nu}$ is

$$
K_{l}(z, w)=(\nu+2 l+1)(1+z \bar{w})^{\nu} F\left(l+\nu+1,-l ; 1 ; \frac{|z-w|^{2}}{\left(1+|z|^{2}\right)\left(1+|w|^{2}\right)}\right) \text {. }
$$

In particular $(l=0)$, the reproducing kernel in $A^{2, \nu}$ is

$$
K_{0}(z, w)=(\nu+1)(1+z \bar{w})^{\nu} \text {. }
$$

REMARK. Note also that if $\nu=0$ then (writing as before $z=\tan \frac{\theta}{2} e^{i \phi}$ )

$$
K_{l}(z, 0)=(2 l+1)(1+z \bar{w})^{\nu} F\left(l+1,-l ; 1 ; \sin ^{2} \frac{\theta}{2}\right)=(2 l+1) P_{l}(\cos \theta)
$$

is the spherical function on $S^{2}=\mathrm{SU}(2) / \mathrm{SO}(2)$, where $P_{l}$ are the Legendre polynomials.

\section{APPLICATIONS TO SPECIAL FUNCTIONS.}

Now we can play some games in the style of Vilenkin's book [12]. Chap. II, dealing with SU(2), is especially relevant for us.

Let us use the above formula (8) in conjunction with relation (6). The result is the formula

$$
\begin{aligned}
& \sum_{n=-l}^{\nu} z^{n} \bar{w}^{n} q_{l n}\left(\frac{|z|^{2}}{1+|z|^{2}}\right) q_{l n}\left(\frac{|w|^{2}}{1+|w|^{2}}\right) /\left\|q_{l n}\right\|^{2}= \\
= & (\nu+2 l+1)(1+z \bar{w})^{\nu} F\left(l+\nu+1,-l ; 1 ;-\frac{|z-w|^{2}}{\left(1+|z|^{2}\right)\left(1+|w|^{2}\right)}\right) .
\end{aligned}
$$

Write $\frac{|z|^{2}}{1+|z|^{2}}=t, \frac{|w|^{2}}{1+|w|^{2}}=s$ and let $\gamma$ be the angle between the vectors $z$ and $w$. Then we can write this as 


$$
\begin{aligned}
& \sum_{n=-l}^{\nu}\left(\frac{t s}{(1-t)(1-s)}\right)^{\frac{1}{2}} e^{2 n \gamma} q_{l n}(t) q_{l n}(s) /\left\|q_{l n}\right\|^{2}= \\
= & (\nu+2 l+1)\left(1+\left(\frac{t s}{(1-t)(1-s)}\right)^{\frac{1}{2}} e^{\imath \gamma}\right)^{\nu} \times \\
& \times F(l+\nu+1,-l ; 1 ;-2(t+s-2 t s-\sqrt{t(1-t) s(1-s)} \cos \gamma)) .
\end{aligned}
$$

We may view the above as the Fourier expansion of the function to the right. Thus we conclude that

$$
\begin{aligned}
& (\nu+2 l+1) \int_{0}^{2 \pi}\left(1+\left(\frac{t s}{(1-t)(1-s)}\right)^{\frac{1}{2}} e^{\imath n \gamma}\right) \times \\
& \times F(l+\nu+1,-l ; 1 ;-(t+s-2 t s-2 \sqrt{t(1-t) s(1-s)} \cos \gamma)) e^{-\imath n \gamma} d \gamma= \\
= & \begin{array}{ll}
\left(\frac{t s}{(1-t)(1-s)}\right)^{\frac{n}{2}} q_{\ln }(t) q_{\ln }(s) /\left\|q_{l n}\right\|^{2} & \text { for }-l \leq n \leq \nu . \\
0 & \text { else }
\end{array}
\end{aligned}
$$

This is a multiplication theorem.

Next we invoke the transvectant (see [4], [15]). It is question of the following bilinear differential expression:

$$
\mathcal{T}_{l}\left(f_{1}, f_{2}\right)=\sum_{k=0}^{l}(-1)^{k}\left(\begin{array}{l}
l \\
k
\end{array}\right) \frac{\partial^{k} f_{1}}{\left(\nu_{1}\right)_{k}} \cdot \frac{\partial^{l-k} f_{2}}{\left(\nu_{2}\right)_{l-k}} \quad\left(\partial=\frac{\partial}{\partial z}\right) .
$$

If we let $f_{1}$ transform with weight $\nu_{1}$ and $f_{2}$ transform with weight $\nu_{2}$, then the transvectant $\mathcal{T}_{l}\left(f_{1}, f_{2}\right)$ transforms with weight $\nu_{1}+\nu_{2}+2 s$.

We can now exhibit an isomorphism between the spaces $A_{l}^{2, \nu}$ and $A^{2, \nu+2 l}$ which respects the $\mathrm{SU}(2)$-action (an "intertwining" map). Namely, we take in (8)

$$
\nu_{1}=-\nu-2 l, \quad \nu_{2}=1, \quad f_{1}=g \in A^{2, \nu+2 l}, f_{2}=K_{-1}(z, z)=\left(1+|z|^{2}\right)^{-1} .
$$

This gives an element $f \in A_{l}^{2, \nu}$ defined by

$$
f=K_{0}^{-1} \mathcal{T}_{l}\left(f, K_{0}\right)=(-1)^{l} \sum_{k=0}^{l}(-1)^{k}\left(\begin{array}{l}
l \\
k
\end{array}\right) \frac{g^{(k)}}{(-\nu-2 l)_{k}}\left(\frac{\bar{z}}{1+|z|^{2}}\right)^{l-k} .
$$

To see that the map $g \mapsto f$ indeed is an isomorphism we take $g=z^{n+l},-l \leq n \leq \nu+l$. This gives us, apart from a factor, back the basis vector $e_{n l}$ (use Lemma 4):

$$
\begin{aligned}
f & =(-1)^{l} \sum_{k=0}^{l} \frac{(-l)_{l-k}}{(l-k) !} \frac{(n+l)_{k}^{-}}{(-\nu-2 l)_{k}} z^{n}\left(\frac{z \bar{z}}{1+|z|^{2}}\right)^{l-k}= \\
& =\sum_{k=0}^{l} \frac{(-l)_{l-k}(l+\nu+1)_{l-k}(n+l)_{l}}{(l-k) !(n+1)_{l-k}(-\nu-2 l)_{l}} z^{n}\left(\frac{z \bar{z}}{1+|z|^{2}}\right)^{l-k}= \\
& =\frac{(n+l)_{l}}{(n+1)_{l}(-\nu-2 l)_{l}} z^{n} q_{l n}\left(\frac{|z|^{2}}{1+|z|^{2}}\right)=\frac{(n+l)_{l}}{(n+1)_{l}(-\nu-2 l)_{l}} e_{n l}(z) .
\end{aligned}
$$

Let $\left\{t_{n m}(\xi)\right\}$ be the matrix of action $U_{\xi}^{(\nu+2 l)}$ on the space $A^{2, \nu+2 l}$ in terms of the basis $\left\{z^{n+l}\right\}$, $-l<n<n-l$, i. e.

$$
U_{\xi}^{(\nu+2 l)} z^{n+l} /\left\|z^{n+l}\right\|=\sum_{m=-l}^{n+l} t_{n m}(\xi) z^{m+l} /\left\|z^{m+l}\right\|
$$

The functions $t_{n m}(\xi)$ are expressible in terms of Legendre functions (cf. [12]). Using the isomorphism constructed above we see that the action of $U_{\xi}^{(\nu)}$ on $A_{l}^{2, \nu}$ has the same matrix: 


$$
U_{\xi}^{(\nu+2 l)} e_{n l} /\left\|e_{n l}\right\|=\sum_{m=-l}^{n+l} t_{n m}(\xi) e_{m l} /\left\|e_{m l}\right\| .
$$

If we spell out this in detail, we obtain a relation involving hypergeometric polynomials.

\section{HARMONIC ANALYSIS OF OPERATORS.}

However, the principal goal of all this business is operator theory. Now we say a few words what operator consequences can be drawn from our results.

We are interested in linear operators acting between eigenspaces $A_{l}^{2, \nu}$ for different values of $l$ and $\nu$ (or complex conjugates of such spaces). In the former case we are dealing with "generalized Toeplitz operators", in the latter case with "generalized Hankel forms" (we identify an operator from $A_{l l^{\prime}}^{2, \nu^{\prime}}$, say, into $\overline{A_{l}^{2, \nu}}$ with the corresponding bilinear form on the product $A_{l \prime^{\prime}}^{2, \nu^{\prime}} \times A_{l}^{2, \nu}$ ). So it might be justified to use with Nikol'skir the ackronym "Ha-plitz" as a unifying concept.

As the spaces involved all are finite dimensional, everything blows down to linear algebra (matrix theory) or - on a more sophisticated level - representation theory for compact groups. So, at least in principle, the problem is in a way trivial. We remark however that non-trivial analysis problems perhaps arise if we allow the parameters $l$ and/or $\nu$ tend to infinity. If we recall the isomorphism $A_{l}^{2, \nu} \approx A^{2, \nu+2 l}$ (Section 3 ), we see also that we have essentially the classical problem connected with the determination of Clebsch-Gordan coefficients in disguise (see e. $\mathrm{g}$. [12], $\S 8$ of Chap. 3).

We begin with the observation that it is, in principle, superfluous to consider conjugate spaces. Indeed, there is a canonical isomorphism from $A^{2, \nu}$ onto $\overline{A^{2, \nu}}$, which is given by

$$
f(z) \mapsto g(z) \equiv \bar{z}^{\nu} f\left(\frac{1}{\bar{z}}\right)
$$

and which intertwines with the group action.

Let us check the intertwining property: If $f$ is replaced by $f\left(\frac{a z+b}{c z+d}\right)(c z+d)^{\nu}$, then $g(z)$ gets replaced by

$$
\begin{aligned}
& \bar{z}^{\nu} f\left(\frac{a \frac{1}{\bar{z}}+b}{c \frac{1}{\bar{z}}+d}\right)\left(c \frac{1}{\bar{z}}+d\right)^{\nu}= \\
= & \left(\overline{\frac{a z+b}{c z+d}}\right)^{\nu} f\left(\overline{\frac{c z+d}{a z+b}}\right)(\overline{c z+d})^{\nu}=g\left(\frac{a z+b}{c z+d}\right)(\overline{c z+d})^{\nu} .
\end{aligned}
$$

So in a way the distinction between Hankel and Toeplitz fades away ...

Let us now look at linear operators from $A^{2, \nu^{\prime}}$ into $A^{2, \nu}$. Assume, to fix the ideas, that $\nu^{\prime} \leq \nu$. Then the simplest SU(2)-invariant operator from $A^{2, \nu^{\prime}}$ into $A^{2, \nu}$ is the Toeplitz type operator $T_{B}$ with kernel

$$
T_{B}(z, w)=f(z)(1+z \bar{w})^{\nu^{\prime}}
$$

where the "symbol" $B$ is a polynomial of degree $\leq t$ which transforms according to the representation $U^{(t)}$, that is, like a form of degree $-\frac{t}{2}$. (If $\nu=\nu^{\prime}$ we get a Toeplitz operator exactly (multiplication by the symbol).) It will be convenient to refer to the number $-t$ as the weight. We shall assume that $t \leq \nu+\nu^{\prime}$. (This will be explained in a more general context below, see (17).)

In order for the kernel in (10) to have the right transformation properties, that is, to be an element of the tensor product $A^{2, \nu} \otimes \overline{A^{2, \nu^{\prime}}}$ we must have

$$
\nu=t+\nu^{\prime}
$$

Indeed, the kernel $(1+z \bar{w})^{\nu^{\prime}}$ behaves as an element of $A^{2, \nu^{\prime}} \otimes \overline{A^{2, \nu^{\prime}}}$ (of "biweight" $\left(-\nu^{\prime},-\nu^{\prime}\right)$ ). So it is clear that $T_{f}(z, w)$ has the right behavior in the variable $w$, this even irrespective of how $f$ transforms. But, by the same reason, in the variable $z$ the product in (10) transforms then with weight $t+\nu^{\prime}$, establishing (11). 
As a generalization of (10), we may, following the procedure in [15], consider for an integer $s$ the operators $T_{B}^{(t)}$ with kernel

$$
T_{B}^{(t)}(z, w)=(1+z \bar{w})^{s}\left(\frac{\partial}{\partial z}\right)^{\sigma}\left[B(z)(1+z \bar{w})^{\nu^{\prime}-s}\right]
$$

where we again assume that the symbol $B$ transforms with weight $-t$. If $\sigma=0$, (12) reduces to (10). In other words: $T_{B}=T_{B}^{\left(\nu-\nu^{\prime}\right)}$. The connection between $\sigma, s$ and $t$ in general will be uncovered now.

As there is no differentation in the $w$ variable it is again trivial that we have the correct $w$ behavior. We require that the product with brackets in (12) be of weight $1-\sigma$. This gives the relation

$$
s-t-\nu^{\prime}=1-\sigma \text { or } \sigma+s=t+\nu^{\prime}+1 .
$$

If we apply "Bol's lemma" (see [4]) it follows that, after the differentation carried out, we have an expression whose weigth is $1+\sigma$. For the result to have the required weight $-\nu$ we then get an equation which we may write as

$$
s-\nu=1+\sigma \text { or } \sigma-s=-\nu-1 .
$$

Thus, eliminating $s$ between (13) and (14), we get

$$
t=\nu-\nu^{\prime}+2 \sigma \quad \text { or } \quad \sigma=\frac{t-\left(\nu-\nu^{\prime}\right)}{2}
$$

which is analogous to the corresponding relation in [15]. ${ }^{4}$ Clearly, (15) implies that

$$
t \geq \nu-\nu^{\prime}
$$

( $\sigma$ must be a positive number), which generalizes (11), and further that $t \equiv \nu-\nu^{\prime} \bmod 2$. From (16) we can likewise determine $s$ :

$$
s=1+\nu+\sigma=1+\nu+\frac{t-\left(\nu-\nu^{\prime}\right)}{2}=1+\frac{\nu+\nu^{\prime}+t}{2},
$$

so both $s$ and $\sigma$ are now expressed in terms of $t .^{5}$ If $\sigma=0,(15)$ reduces to (11) and then $s=1+\nu$.

Besides (16) there is one more inequality imposed on $t$. Namely:

$$
t \leq \nu+\nu^{\prime}
$$

To see this, we observe that if we differentiate the factor $(1+z \bar{w})^{\nu^{\prime}-s}$ the exponent goes down to $\nu^{\prime}-s-\sigma$. From this we obtain $\nu^{\prime}-\sigma \geq 0$, which is in view of (15) the same as (17).

Thus altogether we have $((16)+(17))$

$$
\nu-\nu^{\prime} \leq t \leq \nu+\nu^{\prime} \text {. }
$$

This agrees with the classical fact that

$$
A^{2, \nu} \otimes A^{2, \nu^{\prime}} \approx \sum^{\oplus} A^{2, t}
$$

where we sum over precisely the indices $t$ occuring in (1) with the same parity as the difference $\nu-\nu^{\prime}$ (see [12], page 177). Thus we have made this decomposition rather explicit. Note that the range of $\sigma$ is the interval $\left[0, \nu^{\prime}\right]$.

Let us indicate one application of the preceding connected with Clebsch-Gordan coefficients. We take the symbol to be a monomial, $B(z)=z^{k}(k=0,1, \ldots, t)$, and apply the corresponding operator to a monomial. We observe first that the kernel satisfies

$$
T_{z^{k}}^{(t)}\left(e^{i \phi} z, e^{i \phi} w\right)=e^{i(k-\sigma) \phi} T_{z^{k}}^{(t)}(z, w)
$$


It follows that the operator intertwines with the $\mathrm{SO}(2)$-action:

$$
U_{\phi} T_{z^{k}}^{(t)} U_{\phi}^{-1}=e^{i(k-\sigma) \phi} T_{z^{k}}^{(t)}
$$

where $U_{\phi} f(z)=f\left(e^{\imath \phi} z\right)$. This again implies that monomials are mapped onto monomials. More precisely, we have

$$
T_{z^{k}}^{(t)} z^{n^{\prime}}=C_{t}\left(n, n^{\prime}\right) z^{n} \quad \text { with } \quad n=n^{\prime}+k-\sigma
$$

where the numbers $C_{t}\left(n, n^{\prime}\right)$ are our version of the Clebsch-Gordan coefficients. (We do not indicate in the notation the dependence on $\nu$ and $\nu^{\prime}$.)

For a general symbol this gives

$$
\hat{T}_{B}^{(t)}\left(n, n^{\prime}\right)=C_{t}\left(n, n^{\prime}\right) \hat{B}\left(n-n^{\prime}+\sigma\right)
$$

where we presently use the hat ^ to denote Fourier coefficients. Now a general operator can, by the above, be decomposed into a sum of Toeplitz type operators:

$$
T=\sum_{t} T_{B_{i}}^{(t)}
$$

It follows that we have the following expression for the Hilbert-Schmidt $\left(S_{2^{-}}\right)$norm:

$$
\|T\|_{S_{2}}^{2}=\sum_{t}\left\|T_{B_{t}}^{(t)}\right\|_{S_{2}}^{2}=\sum_{t} \sum_{n=0}^{\nu} \sum_{n^{\prime}=0}^{\nu^{\prime}}\left|C_{t}\left(n, n^{\prime}\right)\right|^{2}\left\|z^{n}\right\|_{\nu}^{2}\left\|z^{n^{\prime}}\right\|_{\nu^{\prime}}^{2}
$$

In particular, we obtain the following orthogonality relation (cf. [9] for a similar result in the hyperbolic case):

$$
\sum_{n-n^{\prime}=\text { const }} C_{t}\left(n, n^{\prime}\right) \overline{C_{t^{\prime}}\left(n, n^{\prime}\right)}\left\|z^{n}\right\|_{\nu}^{2}\left\|z^{n^{\prime}}\right\|_{\nu^{\prime}}^{2}=0 \quad \text { if } \quad t \neq t^{\prime}
$$

The numbers $C_{t}\left(n, n^{\prime}\right)$ can also be expressed in terms of the generalized hypergeometric func$\operatorname{tion}_{3} F_{2}$ :

PROPOSITION 1. $C_{t}\left(n, n^{\prime}\right)=$ const $\cdot_{3} F_{2}\left(-\sigma, \nu^{\prime}-s,-n^{\prime} ;-\nu^{\prime}, 1+k-\sigma ;-1\right)$.

PROOF: We have (see (12))

$$
\begin{aligned}
T_{z^{k}}^{(t)} z^{n^{\prime}}= & \int_{\mathbf{C}}(1+z \bar{w})^{s}\left(\frac{\partial}{\partial z}\right)^{\sigma}\left[z^{k}(1+z \bar{w})^{\nu^{\prime}-s}\right] w^{n^{\prime}} \frac{d \mathbf{a}(w)}{\left(1+|w|^{2}\right)^{\nu^{\prime}+2}}= \\
= & \int_{\mathbf{C}}(1+z \bar{w})^{s} \sum_{j=0}^{\sigma}\left(\begin{array}{c}
\sigma \\
j
\end{array}\right)(k)_{\sigma-j}^{-} z^{k-\sigma+j}\left(\nu^{\prime}-s\right)_{j}^{-} \times \\
& \times(1+z \bar{w})^{\nu^{\prime}-s} w^{n^{\prime}} \frac{d \mathbf{a}(w)}{\left(1+|w|^{2}\right)^{\nu^{\prime}+2}}= \\
= & \sum_{j=0}^{\sigma}\left(\begin{array}{l}
\sigma \\
j
\end{array}\right)(k)_{\sigma-j}^{-}\left(\nu^{\prime}-s\right)_{j}^{-} z^{k-\sigma+j} \times \\
& \times \int_{\mathbf{C}}(1+z \bar{w})^{\nu^{\prime}-j} \bar{w}^{\jmath} w^{n^{\prime}} \frac{d \mathbf{a}(w)}{\left(1+|w|^{2}\right)^{\nu^{\prime}+2}}=
\end{aligned}
$$




$$
\begin{aligned}
= & \sum_{\jmath=0}^{\sigma}\left(\begin{array}{c}
\sigma \\
j
\end{array}\right)(k)_{\sigma-\jmath}^{-}\left(\nu^{\prime}-s\right)_{\jmath}^{-} z^{k-\sigma+\jmath} \times \\
& \times \sum_{h=0}^{\nu^{\prime}-\jmath}\left(\begin{array}{c}
\nu^{\prime}-j \\
h
\end{array}\right) \int_{\mathbf{C}} z^{h} \bar{w}^{\jmath+h} w^{n^{\prime}} \frac{d \mathbf{a}(w)}{\left(1+|w|^{2}\right)^{\nu^{\prime}+2}} .
\end{aligned}
$$

The integral gives a contribution only if $j+h=n^{\prime}$. In this case it reduces to

$$
\begin{aligned}
\int_{\mathbf{C}} \frac{|w|^{2 n^{\prime}} d \mathbf{a}(w)}{\left(1+|w|^{2}\right)^{\nu^{\prime}+2}} & =\int_{0}^{\infty} \frac{r^{2 n^{\prime}} \cdot 2 \pi r d r}{\pi\left(1+r^{2}\right)^{\nu^{\prime}+2}}=\int_{0}^{\infty} \frac{\rho^{2 n^{\prime}} d \rho}{\left(1+\rho^{2}\right)^{\nu^{\prime}+2}}= \\
& =B\left(n^{\prime}+1, \nu^{\prime}-n^{\prime}+1\right)=\frac{\Gamma\left(n^{\prime}+1\right) \Gamma\left(\nu^{\prime}-n^{\prime}+1\right)}{\Gamma\left(\nu^{\prime}+2\right)}= \\
& =\frac{n^{\prime} !\left(\nu^{\prime}-n^{\prime}\right) !}{\left(\nu^{\prime}+1\right) !}
\end{aligned}
$$

Therefore we obtain

$$
\begin{aligned}
C_{t}\left(n, n^{\prime}\right) & =\frac{n^{\prime} !\left(\nu^{\prime}-n^{\prime}\right) !}{\left(\nu^{\prime}+1\right) !} \sum_{\jmath=0}^{\sigma} \frac{(-1)^{\jmath}(-\sigma)_{\jmath}}{j !}(-1)^{\sigma-\jmath}(-k)_{\sigma-\jmath}(-1)^{\jmath}\left(\nu^{\prime}-s\right)_{\jmath} \frac{\left(\nu^{\prime}-j\right) !}{\left(n^{\prime}-j\right) !\left(\nu^{\prime}-n^{\prime}\right) !}= \\
& =(-1)^{\sigma} \frac{n^{\prime} !}{\left(\nu^{\prime}+1\right) !} \sum_{\jmath=0}^{\sigma} \frac{(-\sigma)_{\jmath}(-1)^{\jmath}(-k)_{\sigma}\left(\nu^{\prime}-s\right)_{\jmath}}{j !(1+k-\sigma)_{\jmath}} \frac{\nu^{\prime} !\left(n^{\prime}\right)_{\jmath}^{-}}{n^{\prime} !\left(\nu^{\prime}\right)_{\jmath}^{-}}= \\
& =\frac{(-1)^{\sigma}(-k)_{\sigma}}{\nu^{\prime}+1} \sum_{\jmath=0}^{\sigma}(-1)^{\jmath} \frac{(-\sigma)_{j}\left(\nu^{\prime}-s\right)_{\jmath}\left(-n^{\prime}\right)_{\jmath}}{j !(1+k-\sigma)_{j}\left(-\nu^{\prime}\right)_{\jmath}}= \\
& =\frac{(-1)^{\sigma}(-k)_{\sigma}}{\nu^{\prime}+1}{ }_{3} F_{2}\left(-\sigma, \nu^{\prime}-s,-n^{\prime} ;-\nu^{\prime}, 1+k-\sigma ;-1\right)
\end{aligned}
$$

and we are done.

Finally, duplicating what is done in [10] and [14] in the hyperbolic case, we say a few words about SU(2)-invariant Ha-plitz operators from $A^{2, \nu}$ into $A_{l}^{2, \nu}$ (it is thus the special case $l^{\prime}=0$, $\nu^{\prime}=\nu$ of the general situation). Such an operator can be defined by the bilinear formula

$$
\left\langle H_{B}^{(l, s)} f, g\right\rangle=\int_{\mathbf{C}} f(z) \overline{B(z) \bar{D}^{s} g(z)} \frac{d \mathbf{a}(z)}{\left(1+|z|^{2}\right)^{\nu^{\prime}+2}} .
$$

(Thus, $B(z)$ is the symbol of the operator $H_{B}^{(l, s)}$.) If $V$ is the isomorphism from $A^{2, \nu+2 l}$ onto $A_{l}^{2, \nu}$ displayed in Section 3, then it is easy to verify (for details of this computation see [14]) that $\left(H_{B}^{(l, s)}\right)^{*} V$, which is thus an operator from $A^{2, \nu+2 l}$ to itself, is one of the Toeplitz like operators $T_{B}^{(t)}$ considered earlier in this Section.

\section{DIFFERENTIAL EQUATIONS SATISFIED BY THE BASIS VECTORS.}

In Section 1 we constructed the basis $\left\{e_{n l}(z)\right\}=\left\{z^{n} q_{l n}\left(\frac{|z|^{2}}{1+|z|^{2}}\right)\right\}$, where $-l \leq n \leq l+\nu$, in the space $L^{2, \nu}\left(S^{2}\right)$. Now we write down various ordinary differential equations connected with these functions.

First of all, as the polynomials $q_{l n}(t)$, for $n$ fixed, are orthogonal in the metric

$$
\|q\|^{2}=\int_{0}^{\infty}|q(t)|^{2} t^{n}(1-t)^{\nu-n} d t
$$

it is easy to see, by general principles governing orthogonal polynomials, that they satisfy the differential equation

$$
-t^{-n}(1-t)^{n-\nu} \frac{d}{d t}\left[t^{n+1}(1-t)^{\nu-n+1} \frac{d q}{d t}\right]=l(\nu+l+1) q
$$

or, carrying out the differentiation, 


$$
-t(1-t) \frac{d^{2} q}{d t^{2}}-[\underbrace{(n+1)(1-t)-(\nu-n+1) t}_{n+1-(\nu+2) t}] \frac{d q}{d t}=l(\nu+l+1) q .
$$

This is of course just a special case of the hypergeometric equation in slight disguise.

On the other hand, we know by their very construction that the functions $e_{n l}(z)$ satisfy the partial differential equation

$$
\left.-\left(1+|z|^{2}\right)^{2} \partial \bar{\partial} f+\nu\left(1+|z|^{2}\right) \bar{z} \bar{\partial} f=l(\nu+l+1) f \quad \text { (notation: } \partial=\frac{\partial}{\partial z}, \bar{\partial}=\frac{\partial}{\partial \bar{z}}\right) .
$$

Of course, separating variables, this has to lead back to the same equation (1). Let us verify this! Differentiating the relation $f(z)=z^{n} q\left(\frac{|z|^{2}}{1+|z|^{2}}\right)$ yields

$$
\begin{aligned}
& \bar{\partial} f=z^{n} q^{\prime} \frac{z}{\left(1+|z|^{2}\right)^{2}}=q^{\prime} \frac{z^{n+1}}{\left(1+|z|^{2}\right)^{2}} \\
& \partial \bar{\partial} f=q^{\prime \prime} \frac{z^{n}|z|^{2}}{\left(1+|z|^{2}\right)^{4}}+q^{\prime} \cdot(n+1) \frac{z^{n}}{\left(1+|z|^{2}\right)^{2}}-2 q^{\prime} \frac{z^{n}|z|^{2}}{\left(1+|z|^{2}\right)^{3}}
\end{aligned}
$$

Adding up and using $t=\frac{|z|^{2}}{1+|z|^{2}}, 1-t=\frac{1}{1+|z|^{2}}$ we find

$$
-\left(1+|z|^{2}\right)^{2} \partial \bar{\partial} f+\nu\left(1+|z|^{2}\right) \bar{z} \bar{\partial} f=\left[-t(1-t) q^{\prime \prime}+(\nu+2) t q^{\prime}-(n+1) q^{\prime}\right] z^{n}
$$

which apparently yields (19).

Let us return for a moment to the "hyperbolic" case (disk or halfplane). Actually even before the paper [10] was ever conceived, the same eigenvalue problem had been studied (unknown to us at the time!) in [3] in a different context (in connection with the Feynman integral "with affine kinetic variables") and there it had essentially been reduced to a single one dimensional Schrödinger (or Sturm-Liouville) equation, namely with the Morse operator

$$
\frac{d^{2}}{d y^{2}}+D\left(e^{-2 y}-e^{-y}\right) \quad(D \text { a constant })
$$

in $L^{2}(\mathbf{R})$. Recall that this operator first appeared as a phenomenological model for a diatomic molecule [7]. ${ }^{6}$ Indeed, this is intimately related to the "continuous" orthogonal basis constructed in $[10]^{7}$, viz.

$$
e^{\imath x \xi} e^{-\xi y} \xi^{\alpha+1-l} P_{l}\left(\frac{1}{2 \xi y}\right) \quad\left(l=0,1, \ldots,\left[\frac{\alpha+1}{2}\right]\right),
$$

where $P_{l}(t)$ are certain orthogonal polynomials considered by Romanovski [11]. They are (see [11]) orthogonal with respect to the weigth $t^{\alpha+2} e^{-\frac{1}{2}}$, are given by a Rodrigues formula

$$
P_{l}(t)=t^{\alpha+2} e^{1 / t}\left(\frac{d}{d t}\right)^{l}\left[t^{2 l-\alpha-2} e^{-1 / t}\right],
$$

and satisfy an ordinary differential equation:

$$
t^{2} P^{\prime \prime}+(1-\alpha t) P^{\prime}=l(l-\alpha-1) P .
$$

It is very curious that essentially the same differential equation in two slightly different guises appeared in the same year $1929^{8}$ and in two completely different fields: in statistics, respectively in quantum theory.

Let us formally work out the connection.

In multiplicative language the Morse operator is given by (see [7])

$$
\mathcal{L} \psi=-\left[\xi^{2} \psi^{\prime \prime}+2 \xi \psi^{\prime}+\left(-\xi^{2}+\nu \xi+\frac{1}{4}\right)\right] \psi+\left(\frac{\alpha+1}{2}\right)^{2} \psi,
$$

where $\nu=\frac{\alpha+2}{2}\left(=\frac{\beta}{2}\right.$ in the notation of [3]). Its eigenvalues are the numbers 


$$
\left(\frac{\alpha+1}{2}\right)^{2}-\left(\frac{\alpha+1}{2}-l\right)^{2}=l(\alpha+1-l) \quad\left(l=0,1, \ldots,\left[\frac{\alpha+1}{2}\right]\right)
$$

Write

$$
\psi(\xi)=P\left(\frac{1}{2 \xi}\right) \xi^{\frac{\alpha}{2}} e^{-\xi}
$$

Differentiating we find

$$
\begin{aligned}
\psi^{\prime} & =P^{\prime}\left(\frac{1}{2 \xi}\right) \frac{-1}{2} \xi^{\frac{\alpha}{2}-2} e^{-\xi}+P\left(\frac{1}{2 \xi}\right)\left(\frac{\alpha}{2} \xi^{\frac{\alpha}{2}-1}-\xi^{\frac{\alpha}{2}}\right) e^{-\xi}, \\
\psi^{\prime \prime}= & P^{\prime \prime}\left(\frac{1}{2 \xi}\right) \frac{1}{4} \xi^{\frac{\alpha}{2}-4} e^{-\xi}+P^{\prime}\left(\frac{1}{2 \xi}\right)\left(-\frac{\frac{\alpha}{2}-2}{2} \xi^{\frac{\alpha}{2}-3}+\frac{1}{2} \xi^{\frac{\alpha}{2}-2}\right) e^{-\xi}+ \\
& +P^{\prime}\left(\frac{1}{2 \xi}\right)\left(-\frac{\alpha}{4} \xi^{\frac{\alpha}{2}-3}+\frac{1}{2} \xi^{\frac{\alpha}{2}-2}\right) e^{-\xi}+ \\
& +P\left(\frac{1}{2 \xi}\right)\left(\frac{\alpha}{2}\left(\frac{\alpha}{2}-1\right) \xi^{\frac{\alpha}{2}-2}-\frac{\alpha}{2} \xi^{\frac{\alpha}{2}-1}-\frac{\alpha}{2} \xi^{\frac{\alpha}{2}-1}+\xi^{\frac{\alpha}{2}}\right) e^{-\xi} \\
= & {\left[P^{\prime \prime}\left(\frac{1}{2 \xi}\right) \frac{1}{4} \xi^{\frac{\alpha}{2}-4}+P^{\prime}\left(\frac{1}{2 \xi}\right)\left(-\left(\frac{\alpha}{2}-1\right) \xi^{\frac{\alpha}{2}-3}+\xi^{\frac{\alpha}{2}-2}\right)+\right.} \\
& \left.+P\left(\frac{1}{2 \xi}\right)\left(\frac{\alpha}{2}\left(\frac{\alpha}{2}-1\right) \xi^{\frac{\alpha}{2}-2}-\alpha \xi^{\frac{\alpha}{2}-1}+\xi^{\frac{\alpha}{2}}\right)\right] e^{-\xi} .
\end{aligned}
$$

Put $t=\frac{1}{2 \xi}$ or $\xi=\frac{1}{2 t}$. It follows that (we omit the factor $\xi^{\frac{\alpha}{2}} \cdot e^{-\xi}$ and write $P$ etc. instead of $\left.P^{\prime}\left(\frac{1}{2 \xi}\right)\right)$

$$
\begin{aligned}
\xi^{2} \psi^{\prime \prime} & =P^{\prime \prime} t^{2}+P^{\prime}\left(-2\left(\frac{\alpha}{2}-1\right) t+1\right)+P\left(\frac{\alpha}{2}\left(\frac{\alpha}{2}-1\right)-\frac{\alpha}{2} t^{-1}+\frac{1}{4} t^{-2}\right) \\
\xi \psi^{\prime} & =-P^{\prime} t+P\left(\frac{\alpha}{2}-\frac{1}{2} t^{-1}\right) \\
\left(-\xi^{2}+\nu \xi+\frac{1}{4}\right) \psi & =P\left(-\frac{1}{4} t^{-2}+\frac{\nu}{2} t^{-1}+\frac{1}{4}\right)
\end{aligned}
$$

Hence

$$
\begin{aligned}
\mathcal{L} \psi= & -\left[P^{\prime \prime} t^{2}+P^{\prime}\left(-2\left(\frac{\alpha}{2}-1\right) t+1-2 t\right)+\right. \\
& \left.+P\left(\frac{\alpha}{2}\left(\frac{\alpha}{2}-1\right)-\frac{\alpha}{2} t^{-1}+\frac{1}{4} t^{-2}+\alpha-t^{-1}-\frac{1}{4} t^{-2}+\frac{\nu}{2} t^{-1}+\frac{1}{4}\right)\right] \\
= & -[P^{\prime \prime} t^{2}+P^{\prime}(1-\alpha t)+P \cdot \underbrace{\frac{\alpha^{2}-2 \alpha+4 \alpha+1}{4}}_{\left(\frac{\alpha+1}{2}\right)^{2}}]+\left(\frac{\alpha+1}{2}\right)^{2} P= \\
= & -\left[P^{\prime \prime}+(1-\alpha t) P^{\prime}\right] .
\end{aligned}
$$

It follows that (20) is indeed a consequence of the eigenvalue equation $\mathcal{L} \psi=l(\alpha+1-l) \psi$.

It is now natural to ask the question: What is the analogue of the Morse operator for the sphere?

To answer this question we must first digress a little.

DIGRESSION: REDUCTION OF A SELF-ADJOINT SECOND ORDER ORDINARY DIFFERENTIAL EQUATIONS TO NORMAL FORM.

Consider a self-adjoint eigenvalue equation of the form

$$
\mathcal{L} u \equiv-w^{-1} \frac{d}{d t}\left[w a \frac{d q}{d t}\right]+b q=\lambda q
$$

in $L^{2}(I, w d x)$, where $I$ is some interval $\subset \mathbf{R}$ and $w$ is a positive weight. Furthermore, we assume that the coefficient $a$ is positive and, similarly, that $b$ is real. So the operator $\mathcal{L}$ is formally selfadjoint and we expect it to be semibounded from below on a suitable domain. We wish to reduce it to the normal form

$$
-f^{\prime \prime}+V f=\lambda f
$$

in $L^{2}(J, d x)$, where $J$ is some other inteval (the case $a=w=1$ ). We will think of $V$ as the "potential".

ANSATZ. $q(x)=m(x) f(\varphi(x))$.

We then have

$$
\|q\|^{2}=\int_{I}|q|^{2} w d x=\int_{I}|f(\varphi)|^{2} m^{2} w d x
$$


If we require this to coincide with

$$
\|f\|^{2}=\int_{J}|f|^{2} d x
$$

we must have $m^{2} w d x=d \varphi(x)=\varphi^{\prime} d x$, which gives the following condition.

Condition I. $m^{2} w=\left|\psi^{\prime}\right|$ or $m=\frac{\left|\varphi^{\prime}\right|^{\frac{1}{2}}}{w^{\frac{1}{2}}}$.

(There are two cases, according to whether $\varphi$ is a sense preserving diffeomorphism or a sense reversing diffeomorphism.) Derivation yields

$$
\begin{aligned}
q^{\prime} & =f^{\prime} \varphi^{\prime} m+f m^{\prime}, \\
\frac{d}{d x}\left(w a q^{\prime}\right) & =f^{\prime \prime} \varphi^{\prime 2} m w a+f^{\prime}\left(\varphi^{\prime} m\right)^{\prime} w a+f^{\prime} \varphi^{\prime} m^{\prime} w a+f m^{\prime \prime} w a+(w a)^{\prime}\left(f^{\prime} \varphi^{\prime} m+f m^{\prime}\right) .
\end{aligned}
$$

Thus we get a second condition.

CONDITION II. $\left(\varphi^{\prime}\right)^{2} a=1$ or $\varphi^{\prime}= \pm \frac{1}{a^{\frac{1}{2}}}, \varphi= \pm \int \frac{d x}{a^{\frac{1}{2}}}$.

In this hypotheses $(I+I I)$, actually, the coefficient of $f^{\prime}$ vanishes:

$$
\begin{aligned}
& \left(\varphi^{\prime} m\right)^{\prime} w a+\varphi^{\prime} m^{\prime} w a+(w a)^{\prime} \varphi^{\prime} m=0 \Leftrightarrow \\
\Leftrightarrow & \left(\text { division with } \varphi^{\prime} m w a\right) \frac{\left(\varphi^{\prime} m\right)^{\prime}}{\varphi^{\prime} m}+\frac{m^{\prime}}{m}+\frac{(w a)^{\prime}}{w a}=0 \Leftrightarrow \\
\Leftrightarrow & \varphi^{\prime} m^{2} w a=\mathrm{const}
\end{aligned}
$$

and the latter is a logical consequence of I \& II. Now we give a look at the coefficient of $f$. This gives, finally,

$$
V=-\frac{\left(m^{\prime} w a\right)^{\prime}}{m w}+b \text {. }
$$

EXAMPLE.The multiplicative Morse operator

$$
\mathcal{L} \psi=-\left(x^{2} \psi^{\prime}\right)^{\prime}+b \psi
$$

In this case $a=x^{2}, w=1$. (The exact form of $b$ is not of interest to us at the moment.) Condition II gives $\psi^{\prime}=\frac{1}{x}, \psi=\log x$, while Condition I gives $m=x^{\frac{1}{2}}$ so $\psi=x^{-\frac{1}{2}} f(\log x)$, which is the transformation on p. 89 of [3] (see formula (3.12)). Thus we find

$$
V=-\frac{\left(-\frac{1}{2} \cdot x^{-\frac{3}{2}} x^{2}\right)^{\prime}}{x^{-\frac{1}{2}}}+b=-\frac{\left(-\frac{1}{2} \cdot x^{-\frac{1}{2}}\right)^{\prime}}{x^{-\frac{1}{2}}}+b=-\frac{1}{4}+b
$$

so we obtain the "additive" Morse operator.

After this digression we return to the case of the sphere. It is convenient to put $p=\nu-n$ (and forget the $\nu$ ), so the equation that interests us is

$$
-t^{-n}(1-t)^{-p} \frac{d}{d t}\left[t^{n+1}(1-t)^{p+1} \frac{d q}{d t}\right]=l(n+p+l+1) q .
$$

Thus it is a case with $a=t(1-t), b=0, w=t^{n}(1-t)^{p}$. From Condition II we get

$$
\left(\varphi^{\prime}\right)^{2}=\frac{1}{t(1-t)}
$$

This time we choose the minus sign:

$$
\varphi^{\prime}=-\frac{1}{\sqrt{t(1-t)}}, \quad \varphi=-\int \frac{d t}{\sqrt{t(1-t)}} .
$$

The integration is performed by means of the substitution $t=\cos ^{2} \frac{\theta}{2}$ : 


$$
\frac{d t}{\sqrt{t(1-t)}}=-\frac{\sin \frac{\theta}{2} \cos 2 \frac{\theta}{2}}{\sqrt{\cos ^{2} \frac{\theta}{2}\left(1-\cos ^{2} \frac{\theta}{2}\right)}} d \theta=d \theta
$$

Thus the parameter $\theta$ is the same as in Section 1. We are definitely on the right track! From Condition I we now get

$$
m=\left(\frac{1}{\sqrt{t(1-t) t^{n}(1-t)^{p}}}\right)^{\frac{1}{2}}=\frac{1}{t^{\frac{n}{2}+\frac{1}{4}}(1-t)^{\frac{p}{2}+\frac{1}{4}}} .
$$

Logarithmic differentation now yields

$$
\begin{aligned}
m^{\prime} & =m \cdot\left[-\left(\frac{n}{2}+\frac{1}{4}\right) \frac{1}{t}+\left(\frac{p}{2}+\frac{1}{4}\right) \frac{1}{1-t}\right]= \\
& =-\left(\frac{n}{2}+\frac{1}{4}\right) \frac{1}{t^{\frac{n}{2}+\frac{1}{4}+1}(1-t)^{\frac{p}{2}+\frac{1}{4}}}+\left(\frac{p}{2}+\frac{1}{4}\right) \frac{1}{t^{\frac{n}{2}+\frac{1}{4}}(1-t)^{\frac{p}{2}+\frac{1}{4}+1}} .
\end{aligned}
$$

Thus we find

$$
\begin{gathered}
m^{\prime} w a=-\left(\frac{n}{2}+\frac{1}{4}\right) t^{\frac{n}{2}-\frac{1}{4}}(1-t)^{\frac{p}{2}+1-\frac{1}{4}}+\left(\frac{p}{2}+\frac{1}{4}\right) t^{\frac{n}{2}+1-\frac{1}{4}}(1-t)^{\frac{p}{2}-\frac{1}{4}} \\
\left(m^{\prime} w a\right)^{\prime}=-\left(\frac{n}{2}+\frac{1}{4}\right)\left(\frac{n}{2}-\frac{1}{4}\right) t^{\frac{n}{2}-1-\frac{1}{4}}(1-t)^{\frac{p}{2}+1-\frac{1}{4}}- \\
-\left(\frac{n}{2}+\frac{1}{4}\right)\left(\frac{p}{2}+1-\frac{1}{4}\right) t^{\frac{n}{2}-\frac{1}{4}}(1-t)^{\frac{p}{2}-\frac{1}{4}}+ \\
+\left(\frac{p}{2}+\frac{1}{4}\right)\left(\frac{n}{2}+1-\frac{1}{4}\right) t^{\frac{n}{2}-\frac{1}{4}}(1-t)^{\frac{p}{2}-\frac{1}{4}}- \\
-\left(\frac{p}{2}+\frac{1}{4}\right)\left(\frac{p}{2}-\frac{1}{4}\right) t^{\frac{n}{2}+1-\frac{1}{4}}(1-t)^{\frac{p}{2}-1-\frac{1}{4}} \\
m w=t^{\frac{n}{2}-\frac{1}{4}}(1-t)^{\frac{p}{2}-\frac{1}{4}}
\end{gathered}
$$

whence finally (see boxed formula)

$$
\begin{aligned}
V=-\frac{\left(m^{\prime} w a\right)^{\prime}}{m w}= & \left(\frac{n}{2}+\frac{1}{4}\right)\left(\frac{n}{2}-\frac{1}{4}\right) \frac{1-t}{t}+\left(\frac{p}{2}+\frac{1}{4}\right)\left(\frac{p}{2}-\frac{1}{4}\right) \frac{t}{1-t}+ \\
& -\left[\left(\frac{p}{2}+\frac{1}{4}\right)\left(\frac{n}{2}-\frac{3}{4}\right)+\left(\frac{n}{2}+\frac{1}{4}\right)\left(\frac{p}{2}-\frac{3}{4}\right)\right]= \\
= & \frac{n^{2}-\frac{1}{4}}{4} \frac{1-t}{t}+\frac{p^{2}-\frac{1}{4}}{4} \frac{t}{1-t}- \\
& -\left[\frac{n p}{4}-\frac{3}{4} \cdot \frac{p}{2}+\frac{1}{4} \cdot \frac{p}{2}-\frac{3}{16}+\frac{n \cdot p}{4}-\frac{3}{4} \cdot \frac{n}{2}+\frac{p}{4} \cdot \frac{p}{2}-\frac{3}{16}\right] .
\end{aligned}
$$

The constant term here can be rewritten as follows:

$$
\begin{aligned}
& \frac{n p}{4}-\frac{3}{4} \cdot \frac{p}{2}+\frac{1}{4} \cdot \frac{p}{2}-\frac{3}{16}+\frac{n \cdot p}{4}-\frac{3}{4} \cdot \frac{n}{2}+\frac{1}{4} \cdot \frac{p}{2}-\frac{3}{16}= \\
= & \frac{n p}{2}-\frac{1}{2} \cdot \frac{n}{2}-\frac{1}{2} \cdot \frac{p}{2}-\frac{3}{8}=\frac{1}{2}\left(n-\frac{1}{2}\right)\left(p-\frac{1}{2}\right)-\frac{1}{2} .
\end{aligned}
$$

Thus we end up with the Schrödinger operator

$$
-\frac{d^{2} f}{d \theta^{2}}+\left(\frac{n^{2}-\frac{1}{4}}{4} \tan ^{2} \frac{\theta}{2}+\frac{p^{2}-\frac{1}{4}}{4} \cot ^{2} \frac{\theta}{2}-\frac{1}{2}\left(n-\frac{1}{2}\right)\left(p-\frac{1}{2}\right)+\frac{1}{2}\right) f=l(n+p+l+1) f,
$$

which is the sought "periodic" analogue of the Morse potential.

We may summarize the above discussion as follows.

PROPOSITION 2. The eigenvalue problem for the invariant Laplace operator for $-\nu$-forms on $S^{2}$ is equivalent, in a sense made precise in the foregoing, to an infinite system of synchronous Schrödinger (or Sturm-Liouville) eigenvalue equations on the interval $\cdot(0, \pi)$.

REMARK. Why is there only one equation in the hyperbolic case? It is because in [10] we used the halfspace realization of non-Euclidean geometry, so we have a "dilation" invariant situation, 
which thus accounts for the "degeneracy". If we were to use the disk realization, we would certianly again get infinitely many equations. (One has only to substitute, in the above formula, the trigonometric functions by their hyperbolic ("alcoholic") counterparts tanh and coth.) We do not know if any of this has any bearing to physics whatsoever.

\section{APPENDIX. FACTORIZATIONS OF CERTAIN DIFFERENTIAL OPERATORS ON A RIEMANN SURFACE.}

We continue the computation in [10], remark in Section 3.

As there, we let $X$ denote a Riemann surface equipped with an Hermitean metric, in terms of a local coordinate $z$ given by $d s^{2}=g(z)|d z|^{2}$. We consider $\nu$-forms locally given in a coordinate neighborhood (with coordinate $z$ ) by $f=f(z)(d z)^{\nu}$. If we make a change of coordinate $(z \mapsto \chi(z))$ then these coefficients experience the change $f(z) \mapsto f(\chi(z))\left(\chi^{\prime}(z)\right)^{\nu}, g(z) \mapsto g(\chi(z))\left|\chi^{\prime}(z)\right|^{2}$. (Notice that the parameter $\nu$ plays in this Section the same rôle as $\nu / 2$ in (the rest of) [10], while compared to the rest of the present paper it is the same as $-\nu / 2$.) We denote by $L^{2, \nu}(X)$ the space of square integrable $\nu$-forms, that is, $f \in L^{2, \nu}\left(S^{2}\right)$ if and only if $\int_{X}|f|^{2} g^{1-\nu}(z) d x d y<\infty$.

Let us write $\partial=\frac{\partial}{\partial z}, \bar{\partial}=\frac{\partial}{\partial \bar{z}}$ (Wirtinger operators). The invariant Cauchy-Riemann operator is given by

$$
\bar{D}=g^{-1} \bar{\partial} .
$$

We further set

$$
D_{\nu}=-\partial+(\nu-1) \frac{\partial g}{g}
$$

It may be viewed as the adjoint of $\bar{D}=\bar{D}_{\nu}$ regarded as an operator from $L^{2, \nu}$ into $L^{2, \nu-1}$. In particular, it maps $L^{2, \nu-1}$ into $L^{2, \nu}$. (In [10] we interpreted $D_{\nu}$ as the "metric" connection on the sheaf of all holomorphic $\nu$-forms.) The corresponding Laplace operator is defined by

$$
\Delta=\Delta_{\nu}=D_{\nu} \bar{D}
$$

Written out it is

$$
\Delta=-g^{-1} \bar{\partial} \partial+\nu g^{-2}(\partial g) \bar{\partial} .
$$

In [10] we had, for some reason, written the factors in a different order. We now explain this discrepancy better.

It is convenient to set $e=\frac{\partial g}{g}(=\partial \log g)$. In this notation $D_{\nu}=-\partial+(\nu-1) e$. Furthermore, we let

$$
K=-2 g^{-1} \bar{\partial} e=-2 g^{-1} \bar{\partial}\left(\frac{\partial g}{g}\right)=-2 g^{-1} \bar{\partial} \partial(\log g) .
$$

be the Gaussian curvature. Then we have the following lemma (in a local coordinate neighborhood).

Lemma. $[\partial, \bar{D}]=-e \bar{D},[\bar{D}, e]=-\frac{1}{2} K$.

Proof: It is clear that

$$
[\partial, \bar{D}]=\left[\partial, g^{-1} \bar{\partial}\right]=\partial\left(g^{-1}\right) \bar{\partial}=-g^{-2}(\partial g) \bar{\partial}=-e \bar{D} .
$$

On the other hand, as by definition $\bar{\partial} e=-\frac{1}{2} K g$, we find

$$
[\bar{D}, e]=\left[g^{-1} \bar{\partial}, e\right]=g^{-1}(\bar{\partial} e)=-\frac{1}{2} K .
$$

Corollary. $\bar{D} D_{\nu+1}=D_{\nu} \bar{D}+\frac{\nu}{2} K$.

Proof: We get

$$
\begin{aligned}
\bar{D} D_{\nu+1} & =\bar{D}(-\partial+\nu e)=-\partial \bar{D}+[\partial, \bar{D}]+\nu e \bar{D}+\nu[\bar{D}, e]= \\
& =-\partial \bar{D}-e \bar{D}+\nu e \bar{D}-\frac{\nu}{2} K=(-\partial+(\nu-1) e) \bar{D}-\frac{\nu}{2} K=D_{\nu} \bar{D}-\frac{\nu}{2} K
\end{aligned}
$$


From now on we assume that we have a metric with constant curvature $K$. We claim that one can define recursively polynomials $\Theta_{l}(T)$ (where the letter $T$ stands for an indeterminate) such that

$$
D_{\nu} D_{\nu-1} \ldots D_{\nu-l} \bar{D}^{l+1}=\Theta_{l}(\Delta) \text {. }
$$

Indeed, if $l=0$ this is just the definition of $\Delta=\Delta_{\nu}$, with $\Theta_{0}(T)=T$.

Assume that $\Theta_{l-1}$ is already defined and multiply the corresponding relation $(l-1$ instead of l!) with $\Delta$ from the right. This gives:

$$
D_{\nu} D_{\nu-1} \ldots D_{\nu-(l-1)} \bar{D}^{l} D_{\nu} \bar{D}=\Theta_{l-1}(\Delta) \Delta .
$$

Consider the operator $\bar{D}^{l} D_{\nu}$. We find using the corollary repeatedly (the first time we apply it with $\nu$ replaced by $\nu-1$, the second time by $\nu-2$, and so forth):

$$
\begin{aligned}
\bar{D}^{l} D_{\nu} & =\bar{D}^{l-1} \bar{D} D_{\nu}=\bar{D}^{l-1}\left(D_{\nu-1} \bar{D}-(\nu-1) \frac{K}{2}\right)= \\
& =\bar{D}^{l-1} D_{\nu-1} \bar{D}-(\nu-1) \frac{K}{2} \bar{D}^{l-1}= \\
& =\bar{D}^{l-2} \bar{D} D_{\nu-1} \bar{D}-(\nu-1) \frac{K}{2} \bar{D}^{l-1}= \\
& =\bar{D}^{l-2}\left(D_{\nu-2} \bar{D}-(\nu-2) \frac{K}{2}\right) \bar{D}-(\nu-1) \frac{K}{2} \bar{D}^{l-1}= \\
& =\bar{D}^{l-2} D_{\nu-2} \bar{D}^{2}-((\nu-1)+(\nu-2)) \frac{K}{2} \bar{D}^{l-1}= \\
& =\cdots=D_{\nu-l} \bar{D}^{l}-((\nu-1)+(\nu-2)+\cdots+(\nu-l)) \frac{K}{2} \bar{D}^{l-1} .
\end{aligned}
$$

As, summing the arithmetic series, $(\nu-1)+(\nu-2)+\cdots+(\nu-l)=\frac{l(2 \nu-1-l)}{2}$, it follows that

$$
\begin{aligned}
\Theta_{l-1}(\Delta) \Delta & =D_{\nu} D_{\nu-1} \ldots D_{\nu-l} \bar{D}^{l+1}-l(2 \nu-1-l) \frac{K}{4} D_{\nu} D_{\nu-1} \ldots D_{\nu-(l-1)} \bar{D}^{l}= \\
& =D_{\nu} D_{\nu-1} \ldots D_{\nu-l} \bar{D}^{l+1}-l(2 \nu-1-l) \frac{K}{4} \Theta_{l-1}(\Delta) .
\end{aligned}
$$

In other words, we can take

$$
\Theta_{l}(T)=\left(T+l(2 \nu-1-l) \frac{K}{4}\right) \Theta_{l-1}(T),
$$

or, solving this recursion with initial condition $\Theta_{0}(T)=T$,

$$
\Theta_{l}(T)=T\left(T+(2 \nu-2) \frac{K}{4}\right)\left(T+2(2 \nu-3) \frac{K}{4}\right) \ldots\left(T+l(2 \nu-1-l) \frac{K}{4}\right) .
$$

Altogether, we have now established the following result.

THEOREM 2. We have the following factorization

$$
D_{\nu} D_{\nu-1} \ldots D_{\nu-l} \bar{D}^{l+1}=\Delta\left(\Delta+(2 \nu-2) \frac{K}{4}\right)\left(\Delta+2(2 \nu-3) \frac{K}{4}\right) \ldots\left(\Delta+, l(2 \nu-1-l) \frac{K}{4}\right) .
$$

We conclude with several remarks.

REMARK. Introducing the graded vector space $L=\sum^{\oplus} L^{2, \nu}$, where the summation is over some remainder class of the indices $\nu \bmod \mathbf{Z}$, we can regard the operator $\bar{D}$ as an endomorphism of $L$ and, similarly, we can define an endomorphism $D$ on $L$ extending $D_{\nu}: L^{2, \nu} \rightarrow L^{2, \nu+1}$. Then one can get a more elegant formulation of the above results. In particular, we can write $D_{\nu} D_{\nu-1} \ldots D_{\nu-l} \bar{D}^{l+1}$ more compactly just as $D^{l+1} \bar{D}^{l+1}$. This is in line with how it is done in cohomology theory.

REMARK. To some extent the purported generality of an arbitrary Riemann surface is illusory, as by the uniformization theorem one can reduce oneself to the situation of a simply connected manifold, that is, either the disk, the disk or the ("parabolic") plane. One can then always assume that the metric is given by

$$
g=\frac{1}{\left(1+\frac{K|z|^{2}}{4}\right)^{2}}
$$

It follows that

$$
e(z)=-\frac{K}{4} \cdot \frac{\bar{z}}{1+\frac{K|z|^{2}}{4}} .
$$


Putting $e_{0}(z)=\bar{z} /\left(1+\frac{k|z|^{2}}{4}\right)$, the (second) relation in the lemma can be written $\left[\bar{D}, e_{0}\right]=1$, Thus the operators $\bar{D}$ and (multiplication by) $e_{0}$ formally span an algebra isomorphic to the "Weyl algebra" (see [2]). We do not know what the deeper consequences of this observation are, if any. Footnotes. Note that numbers on the left corner of the following footnotes have
been cited in the text.

1 For this case see [13].
${ }^{2}$ For a unified treatment one could put oneself in the general situation of a Riemannian manifold with constant
Gaussian curvature. Cf. Appendix.

${ }^{3}$ If one of the parameters $a, b$ of that formula is a negative integer, then the coefficient of the second term to the right vanishes, so we get

$$
F(a, b ; c ; z)=\frac{\Gamma(c) \Gamma(c-a-b)}{\Gamma(c-a) \Gamma(c-b)} F(a, b ; a+b-c+1 ; 1-z) .
$$

${ }^{4}$ There we wrote, for some reason, $2 t$ in place of $t$.

${ }^{5}$ In [15] the parameter analogous to $s$ was used to label the operator. In retrospect, we see that this was perhaps not the most natural choice.

\footnotetext{
${ }^{6}$ We are greatful to Thierry Paul for acquanting us with the contents of [3], in particular, the rôle of the Morse operator, during a memorable "workshop" at the Mittag-Leffler Institute (fall '90).

${ }^{7}$ In [10] there is also given a "discrete" orthogonal basis, which is the one which is closest to the basis encountered in the present paper (Section 1). In view of what is done in [9] one may also ask what is the analogue of the Morse operator for a strip.

${ }^{8}$ This is also the year of the wedding of the parents of the senior of the two present authors!
}

\section{REFERENCES}

1. BEREZIN, F. A., General concept of quantization. Commun. Math. Phys. 40 (1975), 153174 .

2. BJÖRK, J.-E., Rings of differential operators. North Holland, Amsterdam, 1979.

3. DAUBECHIES, I., KLAUDER, J. and PAUL, T., Wiener measures for path integrals with affine kinetic variables. J. Math. Phys. 28 (1987), 85-102.

4. GUSTAFSSON, B. and PEETRE, J., Notes on projective structures on complex manifolds. Nagoya Math. J. 116 (1989), 63-88.

5. HELGASON, S., Groups and geometric analysis. Academic Press, London, 1984.

6. MAGNUS, W. and OBERHETTINGER, F., Formulas and theorems for the functions of mathematical physics. Chelsea, New York, 1949.

7. P. MORSE, Diatomic molecules according to the wave mechanics. II. Vibrational levels. Phys. Rev. 34 (1929), 57-64.

8. PEETRE, J., The Berezin transform and Ha-plitz operators. J. Oper. Theory 24 (1990), 165-168.

9. PEETRE, J., Orthogonal polynomials arising in connection with Hankel forms of higher weight. Bull. Sci. Math. (2) 116 (1992), H.

10. PEETRE, J., PENG, L. and ZHANG, G., A weighted Plancherel formula I. The case of the unit disk. Applications to Hankel operators. Technical report, Stockholm University, 1990.

11. ROMANOVSKI, M. V., Sur quelques classes nouvelles de polynômes orthogonaux. $C$. $R$. Acad. Sci. Paris 188 (1929), 1023-1025.

12. VILENKIN, N. JA., Fonctions spéciales et théorie de la représentations des groupes. Dunod, Paris, 1969.

13. ZHANG, G., A weighted Plancherel formula II. The case of the ball. Technical report No. 9, Mittag-Leffler Institute, 1990/91.

14. ZHANG, G., Hankel operators between Moebius invariant subspaces. Math. Scand. (to appear).

15. ZHANG, G., Tensor products of weighted Bergman spaces and invariant Ha-plitz operators. Math. Scand. (to appear). 


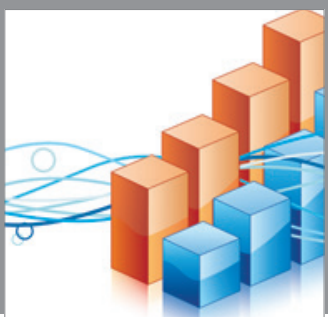

Advances in

Operations Research

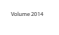

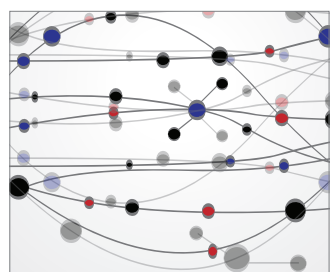

\section{The Scientific} World Journal
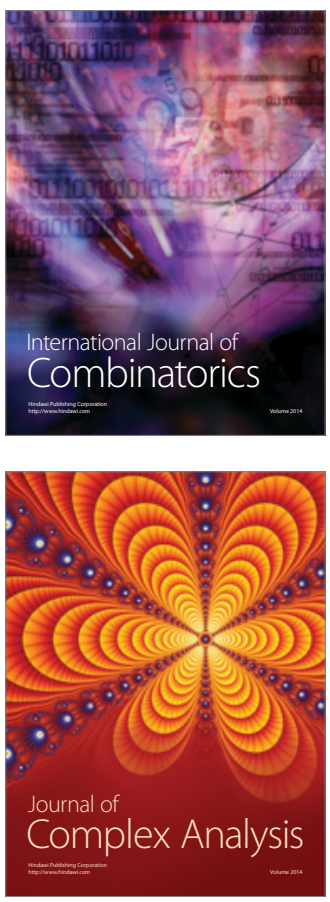

International Journal of

Mathematics and

Mathematical

Sciences
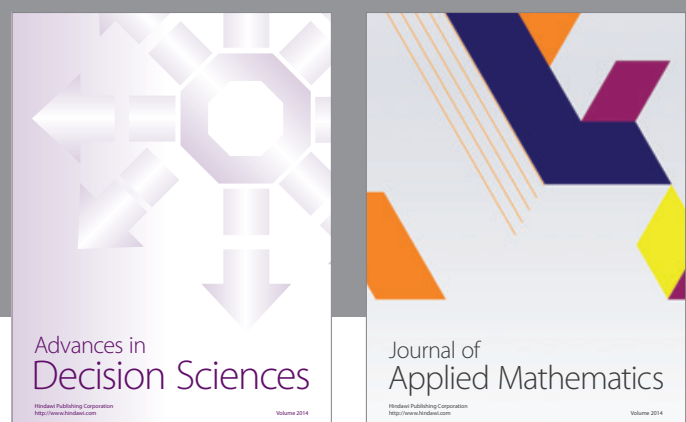

Journal of

Applied Mathematics
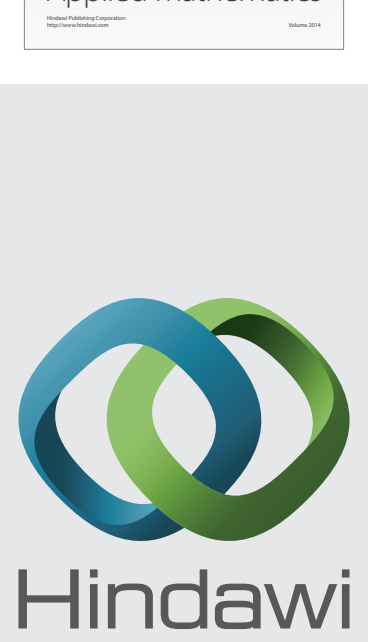

Submit your manuscripts at http://www.hindawi.com
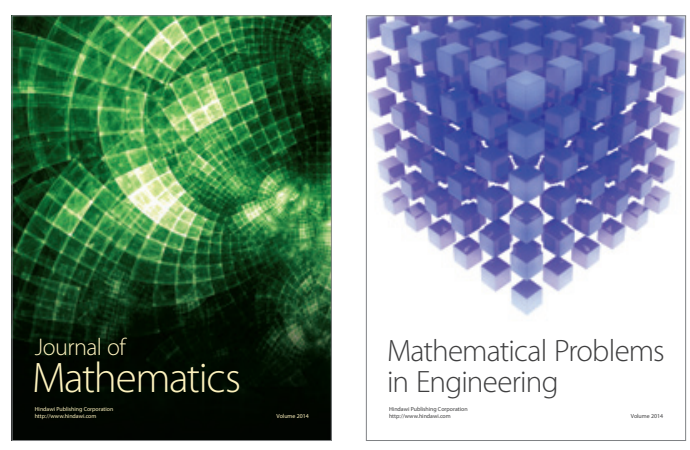

Mathematical Problems in Engineering
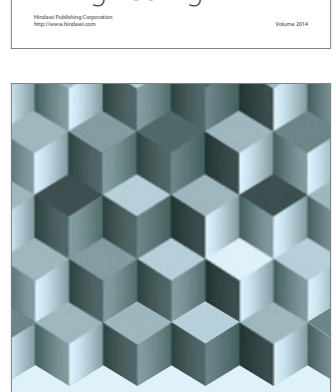

Journal of

Function Spaces
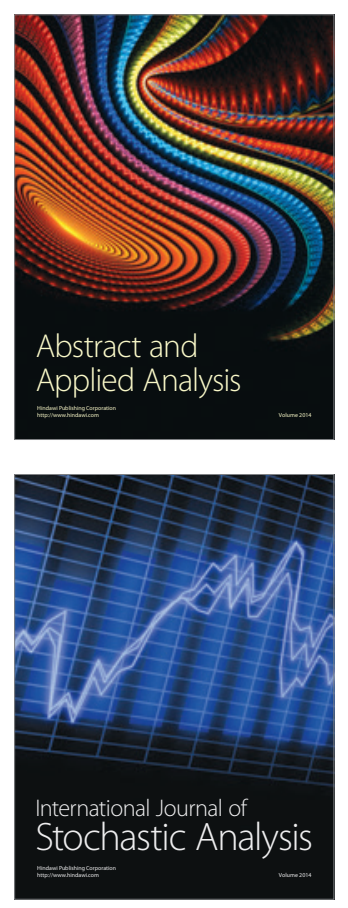

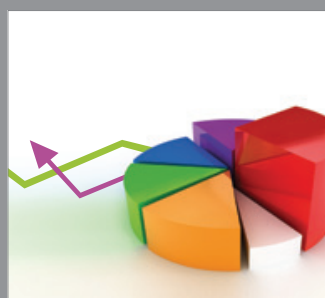

ournal of

Probability and Statistics

Promensencen
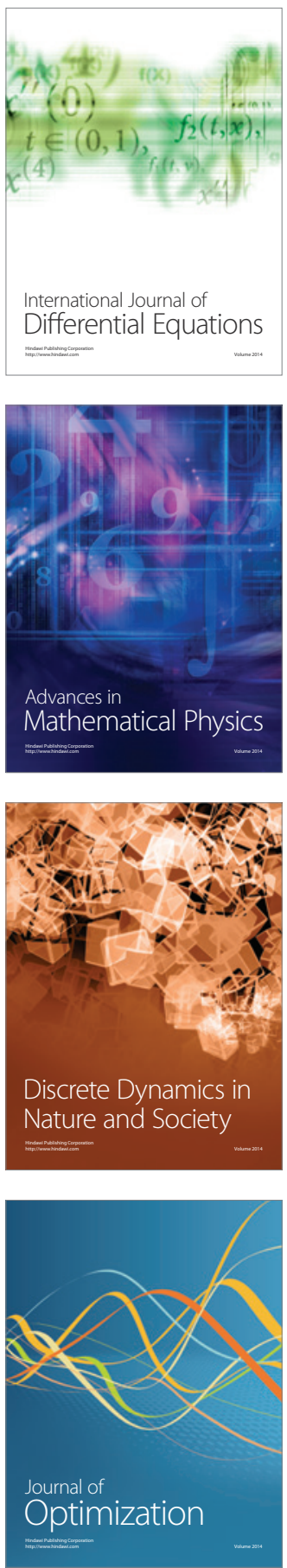